\title{
Inadequacy of the risk factor based approach to detect gestational diabetes mellitus
}

\author{
N J Dahanayaka ${ }^{1}$, S B Agampodi ${ }^{1}$, O R J C Ranasinghe ${ }^{1}$, P M E D Jayaweera ${ }^{1}$, W A N D Wickramasinghe ${ }^{1}$, \\ A N C W B Adhikari ${ }^{1}$, H K J U Chathurani ${ }^{1}$, U T Dissanayaka ${ }^{1}$
}

(Index words: gestational diabetes mellitus, Anuradhapura, Sri Lanka)

\begin{abstract}
Objective To evaluate the present risk factor based approach in diagnosis of gestational diabetes mellitus (GDM) in Sri Lanka in comparison with new guidelines proposed by the International Association of Diabetes and Pregnancy Study Groups (IADPSG).

Methods A community based cross sectional descriptive study was conducted among pregnant women with gestational age of 24-28 weeks and residing in Anuradhapura district. All eligible pregnant women from selected Medical Officers of Health areas were invited to participate. The $75 \mathrm{~g}$ oral glucose tolerance test was carried out among all participants.
\end{abstract}

Results According to IADPSG criteria, 36 (8.9\%) of pregnant women had GDM, compared to 29 (7.2\%) according to WHO criteria. Prevalence of GDM in the study population (positive by one or both methods) was $10.6 \%(n=43)(95 \% \mathrm{Cl} 7.9-13.9 \%)$. Of these 43 women, $22(51.1 \%)$ had positive results by both methods. Seven (16.3\%) and 14 (32.6\%) mothers were positive only according to WHO and IADPSG criteria respectively. Out of 29 mothers who fulfilled WHO criteria, only one had an abnormal fasting plasma glucose, but 28 had abnormal 2 hour values. A total of 170 (42.0\%) participants had at least one risk factor or early indicator of GDM. A risk based approach would have detected only 22 GDM patients according to IADPSG criteria, missing 14 cases (38.9\%) who did not have any of the risk factors for GDM.

Conclusions The risk factor based approach misses more than one third of GDM cases. Urgent revision of current GDM screening guidelines is recommended.

Ceylon Medical Journal 2012; 57: 5-9

\section{Introduction}

Gestational diabetes mellitus (GDM) is defined as any degree of glucose intolerance with first recognition or onset during pregnancy regardless of whether diet modification or insulin is used for treatment, or if the condition persists following pregnancy [1].
Diagnosis of GDM has had many controversies over the past decades. One major reason for lack of consensus was insufficient evidence for treatment and outcome. Two randomised trials provided convincing evidence of positive pregnancy outcomes in treating gestational hyperglycaemia $[2,3]$. Another study showed association of maternal glucose levels below those diagnostic of overt diabetes with adverse maternal and prerinatal outcomes [4]. The International Association of Diabetes and Pregnancy Study Groups (IADPSG) has proposed new diagnostic criteria to diagnose GDM based on the above results. Most professional bodies, including the American Diabetic Association (ADA) changed their guidelines and adopted IADPSG criteria for GDM screening and treatment [5].

The Ministry of Health and Nutrition practice guidelines do not recommend universal screening for GDM in Sri Lanka. Sri Lankan guidelines recommend two hour post prandial glucose (PPBS) after standard meal as the screening test at the booking visit for those with risk factors for GDM. It further states that if PPBS is $\geq 7.2 \mathrm{mmol} /$ $\mathrm{L}$, proceed at once to $75 \mathrm{~g}$ Oral Glucose Tolerance Test (OGTT), and if $<7.2 \mathrm{mmol} / \mathrm{L}$ with at least one risk factor, to proceed to $75 \mathrm{~g}$ OGTT at 24-28 weeks and to diagnose GDM using WHO criteria.

In the light of this new evidence and newly proposed diagnostic criteria, we conducted a study to determine the prevalence of GDM according to IADPSG criteria and to evaluate the present risk factor based approach in diagnosis of GDM in Sri Lanka.

\section{Methods}

The study was conducted in three Medical Officer of Health areas $(\mathrm{MOH})$ in Anuradhapura district: Nuwaragampalatha East, Nuwaragampalatha Central and Kekirawa. These $3 \mathrm{MOH}$ areas have a total population of 270,000 and account for $26.6 \%$ of the total deliveries from Anuradhapura. The study population included all pregnant mothers residing in the selected $\mathrm{MOH}$ areas and receiving services through public health midwives (PHMs). All pregnant mothers completing 24 weeks and not more than 28 weeks of gestation were invited to participate in the study.

${ }^{1}$ Faculty of Medicine and Allied Sciences, Saliyapura, Anuradhapura, Sri Lanka.

Correspondence: NJD, e-mail: <niroshanajd@yahoo.com>. Received 29 August and accepted 22 October 2011. Competing interests: none declared. 
It was calculated that a minimum sample of 135 mothers were needed to detect a 10\% prevalence of GDM with an absolute precision of $5 \%$ in an infinite population. However, the procedure was a consecutive sampling in a finite population and patient recruitment was carried out to cover $10 \%$ of the annual deliveries $(n=400)$ in selected areas. During antenatal clinics and home visits, area PHMs invited all pregnant women with gestational age of 24-28 weeks and residing in selected $\mathrm{MOH}$ areas to participate in the study.

Consenting pregnant women were given clear instructions (verbal and written) on preparation for OGTT and directed to local centres on a day feasible for the participants. During the visits, venous blood samples were obtained for fasting and at $1 \mathrm{hr}$ and $2 \mathrm{hr}$ post glucose load sugar levels. Six MBBS qualified investigators collected data during the 2 hours of waiting period using an interviewer administered questionnaire. Sample collection was carried out by qualified medical laboratory technicians using standard protocols.

Questionnaire was pretested in the same study area but on a separate group of mothers who were not eligible for the study at the time of study proper. Validity and reliability of data were ensured using written protocols, predefined variables, interviewer guidelines, training and supervision. Participant provided data were confirmed using pregnancy records, previous medical records and hospital records. Pregnancy and delivery data were extracted from pregnancy records and confirmed through the questionnaire. Two methods for diagnosis of GDM were used for comparison (Table 1). All laboratory tests were quality controlled and abnormal results were repeated and confirmed. Plasma glucose measurements were carried out with automated analyser using the glucose oxidase enzymatic calorimetric assay.

Data cleaning was done manually and entered directly to a SPSS data base. Prevalence of GDM was first determined using WHO and IADPSG criteria separately. The prevalence was defined as the percentage of women having GDM in at least one set of criteria. To determine the percentage of women that could have been diagnosed if current recommendations were followed, first the mothers with risk factors were selected. These risk factors included previous pregnancy risk factors, present pregnancy risk factors and early indicators of GDM (Table 1). Mothers with a single risk factor for GDM were then examined and classified using WHO criteria. Proportion of GDM diagnosed through this procedure, was compared with the recommended IADPSG guidelines. Approval was obtained from the Ethical Review Committee, Faculty of Medicine and Allied Sciences, Rajarata University of Sri Lanka. Informed, written consent was obtained from all participants.

Table 1. Diagnostic algorithms/definitions used in the present study

\section{GDM based on current recommended practice}

Any mother with one or more of the following risk factors,

Past history of IGT/DM/GDM/PCOD, Age more than 35 years, Weight $>65 \mathrm{~kg}$ or BMI $>25 \mathrm{~kg} / \mathrm{m}^{2}$, First degree relatives with diabetes mellitus, Birth weight $>3.5 \mathrm{~kg}$ in any pregnancy, History of unexplained still births or IUDs, FH>POA, polyhydramnios or macrosomia, recurrent urinary tract infections, candidiasis and $75 \mathrm{~g}$ OGTT results (WHO criteria)

Fasting Plasma Glucose $\geq 7 \mathrm{mmol} / \mathrm{L}$ and/or 2 hour blood glucose $\geq 7.8 \mathrm{mmol} / \mathrm{L}$

\section{GDM based on IADPSG criteria}

Any mother with one or more of the following results in 75g OGTT

Fasting plasma glucose $(\mathrm{FPG}) \geq 5.1 \mathrm{mmol} / \mathrm{L}$

1-h plasma glucose $\geq 10 \mathrm{mmol} / \mathrm{L}$

2-h plasma glucose $\geq 8.5 \mathrm{mmol} / \mathrm{L}$ 
Table 2. Characteristics of 405 pregnant women

\begin{tabular}{|c|c|c|}
\hline & $n$ & $\%$ \\
\hline \multicolumn{3}{|l|}{ Age groups } \\
\hline$\leq 19$ & 32 & 7.9 \\
\hline $20-34$ & 339 & 83.7 \\
\hline$\geq 35$ & 34 & 8.4 \\
\hline \multicolumn{3}{|l|}{ Parity } \\
\hline 1 & 171 & 42.2 \\
\hline 2 & 117 & 28.9 \\
\hline 3 & 82 & 20.2 \\
\hline 4 & 27 & 6.7 \\
\hline 5 or more & 8 & 2.0 \\
\hline \multicolumn{3}{|c|}{$\begin{array}{l}\text { Gestational age at OGTT } \\
\text { performed }\end{array}$} \\
\hline 24-28 weeks & 330 & 81.5 \\
\hline 29-32 weeks & 72 & 17.8 \\
\hline >32 weeks & 3 & 0.7 \\
\hline \multicolumn{3}{|c|}{ Gestational age at registration } \\
\hline$\leq 8$ weeks & 232 & 57.3 \\
\hline 9-12 weeks & 134 & 33.1 \\
\hline >12 weeks & 39 & 9.6 \\
\hline
\end{tabular}

Table 3. Prevalence of GDM according to diagnostic criteria

\begin{tabular}{lrl}
\hline Diagnosis criteria & $n$ & $\%$ \\
\hline WHO & & \\
FPG only $(\geq 7 \mathrm{mmol} / \mathrm{L})$ & 0 & - \\
2 h glucose only $(\geq 7.8 \mathrm{mmol} / \mathrm{L})$ & 28 & 6.91 \\
Both & 1 & 0.25 \\
Total & $\mathbf{2 9}$ & $\mathbf{7 . 1 6}$ \\
& & \\
IADPSG & & \\
FPG only $(\geq 5.1 \mathrm{mmol} / \mathrm{L})$ & 19 & 4.69 \\
1 h glucose only $(\geq 10.0 \mathrm{mmol} / \mathrm{L})$ & 0 & - \\
$2 \mathrm{~h}$ glucose only $(\geq 8.5 \mathrm{mmol} / \mathrm{L})$ & 3 & 0.74 \\
FPG and $1 \mathrm{~h}$ & 4 & 0.99 \\
FPG and $2 \mathrm{~h}$ & 0 & - \\
All three & 3 & 0.74 \\
1h and $2 \mathrm{~h}$ & 7 & 1.73 \\
Total & 36 & $\mathbf{8 . 8 9}$ \\
\hline
\end{tabular}

Table 4. Prevalence of risk factors for GDM among 405 pregnant women

\begin{tabular}{lcc}
\hline Risk factor/ predictor* & $n$ & $\%$ \\
\hline Weight $>65$ kg or BMI $>25 \mathrm{~kg} / \mathrm{m}^{2}$ & 78 & 19.3 \\
First degree relatives with diabetes mellitus & 76 & 18.8 \\
Age more than 35 years & 34 & 8.4 \\
Fundal height $>$ POA & 29 & 7.2 \\
Macrosomia or Polyhydramniosis & 6 & 1.3 \\
Past history of IGT/DM/PCOD & 4 & 1 \\
*Previous pregnancy risk factors (n=235) & & \\
Birth weight >3.5 Kg in any pregnancy & 26 & 11.1 \\
History PIH in any pregnancy & 7 & 3.0 \\
History of unexplained still births or IUDs & 3 & 1.3 \\
GDM in any pregnancy & 1 & 0.4 \\
\hline
\end{tabular}

*primiparous mothers were excluded from the denominator 
Table 5. GDM screening/ diagnostic methods used during first 28 weeks of gestation

\begin{tabular}{|c|c|c|c|c|c|c|c|c|}
\hline \multirow{3}{*}{ Screening test } & \multicolumn{3}{|c|}{ Tested } & \multirow[b]{3}{*}{$n$} & \multicolumn{4}{|c|}{ OGTT } \\
\hline & \multirow[b]{2}{*}{$n$} & \multirow[b]{2}{*}{$(\%)$} & \multirow[b]{2}{*}{ Test results } & & \multicolumn{2}{|c|}{ Normal } & \multicolumn{2}{|c|}{ Abnormal } \\
\hline & & & & & $n$ & $\%$ & $n$ & $\%$ \\
\hline \multirow[t]{2}{*}{ Urine dip stick test } & 389 & (96.0) & Normal & 379 & 340 & 89.4 & 39 & 10.6 \\
\hline & & & Abnormal & 10 & 7 & 70.0 & 3 & 30.0 \\
\hline \multirow[t]{2}{*}{ FPG } & 16 & (4.0) & $<5.1 \mathrm{mmol} / \mathrm{L}$ & 14 & 11 & 78.6 & 3 & 21.4 \\
\hline & & & $\geq 5.1 \mathrm{mmol} / \mathrm{L}$ & 2 & 2 & 100.0 & 0 & 0.0 \\
\hline \multirow[t]{2}{*}{ PPBS } & 113 & (27.9) & $<7.8 \mathrm{mmol} / \mathrm{L}$ & 111 & 94 & 84.7 & 17 & 15.3 \\
\hline & & & $\geq 7.8 \mathrm{mmol} / \mathrm{L}$ & 2 & 0 & 0.0 & 2 & 100.0 \\
\hline GCT & 8 & (2.0) & $\geq 7.8 \mathrm{mmol} / \mathrm{L}$ & 8 & 8 & 100.0 & 0 & - \\
\hline
\end{tabular}

\section{Results}

A total of 405 pregnant women from 61 PHM areas participated in the study. Mean age of the pregnant women was 27.3 years (SD 5.4 years). Primiparous women accounted for $42.2 \%(n=171)$ of the study sample (Table 2). Period of amenorrhoea at registration was less than 12 weeks among $90.3 \%(n=366)$ of the pregnant women. $81.5 \%$ $(n=330)$ of participants were between 24 to 28 weeks of gestation. There were 74 (18.5\%) pregnant mothers who were invited at 28 weeks, but visited the sample collection centres few weeks later.

Median (IQ range), fasting, 1 and 2 hour glucose values on OGTT were 78 (73-83), 123 (103-145), and 105 (91-115) mg/dl, respectively. According to IADPSG criteria, 36 (8.9\%) pregnant women had GDM, compared with 29 (7.2\%) according to WHO criteria. IADPSG criteria for GDM were satisfied in 26 women on Fasting Plasma Glucose (FPG), another seven at $1 \mathrm{~h}$, and three more at $2 \mathrm{~h}$ (Table 3). There were 19 women (3.7\%) with a FPG diagnostic for GDM according to IADPSG criteria, but with normal 1 and $2 \mathrm{~h}$ glucose levels. All seven women with $1 \mathrm{~h}$ abnormal values also had $2 \mathrm{~h}$ abnormal values. WHO criteria for GDM were satisfied in only a single woman on FPG and 28 at $2 \mathrm{~h}$. Prevalence of GDM in the study population (positive in one or both methods) was $10.6 \%(n=43)$ (95\% CI 7.9-13.9\%). Of these 43 women, $22(51.1 \%)$ had positive results in both methods. Seven (16.3\%) women were positive only according to WHO criteria and 14 (32.6\%) were positive only according to IADPSG criteria.

Of the risk factors and early indicators of GDM assessed, 78 (19.3\%) had weight $>65 \mathrm{~kg}$ or BMI $>25 \mathrm{~kg} / \mathrm{m}^{2}$ at booking visit and 76 (18.8\%) reported as having first degree relatives with diabetes mellitus (Table 4). A total of $170(42.0 \%)$ participants had at least one indicator to undergo OGTT at 24 weeks. Of the 170 risk mothers, WHO criteria for GDM were satisfied in a single woman on FPG and 21 at 2 h detecting only $75.9 \%$ of the GDM cases that were detected by universal screening using the same WHO criteria. Compared to IADPSG criteria, the current risk factor approach missed 14 cases (38.9\%) of GDM who did not have any of the risk factors or indicators of GDM.

We retrospectively evaluated screening/ diagnostic methods used to detect GDM during the first trimester to assess the utility of this procedure (Table 5). Urine dip stick test was regularly carried out among 389 (96.0\%) of pregnant women. PPBS was carried out in 113 participants. These 113 included 56 (49.6\%) women with risk factors and 57 (50.4\%) without risk factors. Most (66.4\%) of the PPBS was carried out in a single $\mathrm{MOH}$ area. Two PPBS results showed $\geq 7.2 \mathrm{mmol} / \mathrm{L}$, both were confirmed as GDM in the present study. Of the 10 women who had abnormal urine dipstick results three (30\%) were confirmed of having GDM.

\section{Discussion}

Overt diabetes is known to cause adverse perinatal and maternal outcomes [6]. However, the association between less severe hyperglycaemia and adverse outcomes in pregnancy was not established until recently. Based on these results IADPSG suggested new diagnostic criteria to diagnose and treat GDM which is now adapted in most developed countries [7].

In our study population, 10.6 (95\% CI 7.9-13.9\%) of the pregnant mothers fulfilled at least a single criterion to diagnose GDM. Prevalence of GDM was 8.9\% (95\% CI $6.4-11.0 \%$ ) according to the IADPSG criteria. Previous studies conducted in Sri Lanka only considered WHO diagnostic criteria to diagnose GDM. In our study population prevalence of GDM was 7.2\% (95\% CI 
4.9-10.0\%) based only on WHO criteria whereas it was as high as $10.3 \%$ in a community based study conducted in Homagama in 2003 [8]. However, this is the first community based survey conducted outside Colombo district to determine the prevalence of GDM.

The difference of GDM women captured by IADPSG criteria compared to WHO criteria (assuming universal screening in both methods) in our population was $19.5 \%$. Most of the studies that compared previous guidelines and IADPSG criteria showed higher percentage increase in GDM prevalence with new guidelines based on IADPSG criteria. When it compared to ADA guidelines, 3.6 fold and 2.9 fold increase of prevalence was reported previously $[9,10]$. In Japan change of guidelines expected to increase the prevalence by 2.7 fold [11]. This increased prevalence is mainly attributed to the reduction of threshold levels in FPG to $5.1 \mathrm{mmol} / \mathrm{L}$ [12].

The Sri Lankan guidelines only recommend risk factor based screening for GDM though women of south Asian origin are known to have shown to be having high risk for GDM compared to other ethnic groups. Even though American Association of Obstetricians also recommends a risk based approach, all pregnant women of Asian origin are screened according to their guidelines, based on high genetic susceptibility of Asian women [8]. However, HAPO study findings and evidence from two randomised trials show benefits of treating hyperglycemia and universal screening. Our results showed that risk factor based screening would have missed $38.8 \%$ of cases of GDM in our study population. Similar results were noted in a study conducted at the Castle Street Hospital for Women in Colombo in 2001 where the comparison was done for universal screening and risk based approach using WHO criteria [13].

The time has come to re-evaluate the current GDM screening guidelines of Sri Lanka especially with improving specialist services and basic laboratory facilities in the rural areas. Universal screening for GDM needs to be implemented as risk factor based screening has been repeatedly shown to miss nearly $40 \%$ of the pregnant women with GDM in Sri Lanka. Though it is difficult to establish universal screening procedures overnight, previous studies showed that capillary glucose measurements could be used efficiently as an alternative to venous blood measurements in resource poor settings [14]. Our study did not detect a single case based only on 1h glucose measurement in OGTT. This observation may simplify the screening procedure by using FPG and $2 \mathrm{~h}$ glucose measurements as previously suggested by WHO. Analysis of screening procedures during the first trimester in this study showed that the screening for GDM was carried out in an adhoc manner in the study area. Changes should be effected not only in policy and screening guidelines, but also in providing technical support, guidance and supervision to make sure the recommended practices are properly implemented in peripheral areas where services are rendered with minimal resources and poor facilities.

\section{Acknowledgments}

We acknowledge public health staff in selected $\mathrm{MOH}$ areas, MOMCH and RDHS Anuradhapura for their support throughout the study. This study was funded by Maternal Health Task Force of Engender Health.

\section{References}

1. World Health Organization. Definition, Diagnosis and Classification of Diabetes Mellitus and its Complications. Geneva: Department of Noncommunicable Disease Surveillance, World Health Organization, 1999.

2. Crowther CA, Hiller JE, Moss JR, et al. Effect of treatment of gestational diabetes mellitus on pregnancy outcomes. New England Journal of Medicine 2005; 352: 2477-86.

3. Landon MB, Spong CY, Thom E, et al. A multicentre, randomised trial of treatment for mild gestational diabetes. New England Journal of Medicine 2009; 361: 1339-48.

4. Hyperglycaemia and Adverse Pregnancy Outcome (HAPO) Study: associations with neonatal anthropometrics. Diabetes 2009; 58: 453-9.

5. Standards of medical care in diabetes - 2011. Diabetes Care 2011; 34: 11-61.

6. Chung CS, Myrianthopoulos NC. Factors affecting risks of congenital malformations II. Effect of maternal diabetes on congenital malformations. Birth Defects 1975; 11: 23-38.

7. International Association of Diabetes Pregnancy Study Groups Consensus Panel, International Association of Diabetes and Pregnancy Study Groups Recommendations on the Diagnosis and Classification of Hyperglycemia in Pregnancy. Diabetes Care 2010; 33: 676-82.

8. Ginige S, Wijewardhana K, Wijeyaratne CN. Prevalence of gestational diabetes mellitus in Homagama divisional director of health services area. Journal of the College of Community Physicians of Sri Lanka 2004; 9: 40-2.

9. Mahdavian M, Hivert M-F, Baillargeon J-P, et al. Gestational diabetes mellitus: simplifying the international association of diabetes and pregnancy diagnostic algorithm using fasting plasma glucose. Diabetes Care 2010; 33: e145.

10. Agarwal MM, Dhatt GS, Shah SM. Gestational diabetes mellitus: simplifying the international association of diabetes and pregnancy diagnostic algorithm using fasting plasma glucose. Diabetes Care 2010; 33: 2018-20.

11. Morikawa M, Yamada T, Akaishi R, et al. Change in the number of patients after the adoption of IADPSG criteria for hyperglycaemia during pregnancy in Japanese women. Diabetes Research and Clinical Practice 2010; 90: 339-42.

12. Moses RG, Morris GJ, Petocz P, San Gil F, Garg D. The impact of potential new diagnostic criteria on the prevalence of gestational diabetes mellitus in Australia. Medical Journal of Australia 2011; 194: 338-40.

13. Wagaarachchi PT, Fernando L, Premachandra P, Fernando DJ. Screening based on risk factors for gestational diabetes in an Asian population. Journal of Obstetrics and Gynecology 2001; 21: 32-4.

14. Wijeyaratne CN, Ginige S, Arasalingam A, Egodage C, Wijewardhana K. Screening for gestational diabetes mellitus: the Sri Lankan experience. Ceylon Medical Journal 2006; 51: 53-8. 